\title{
High-Throughput Transcription-mediated amplification (TMA), a real and consolidated alternative for the diagnosis of SARS-COV2. Review.
}

FRANCISCO JAVIER GARCÍA-FERNÁNDEZ ( $\sim$ jgardez@hotmail.com )

Hospital General de Tomelloso

\section{Systematic Review}

Keywords: SARS-COV-2, COVID-19, TMA (High-Throughput Transcription-mediated amplification), RT-PCR

Posted Date: July 22nd, 2021

DOI: https://doi.org/10.21203/rs.3.rs-742440/v1

License: (c) (i) This work is licensed under a Creative Commons Attribution 4.0 International License.

Read Full License 


\section{Abstract}

The exceptional situation in which we have found ourselves since the first case of severe acute respiratory syndrome coronavirus 2 (SARS-CoV-2) was identified, which rapidly evolved into a global pandemic, translated into the need to incorporate the largest number of diagnostic tests to detect possible cases and contain the spread of the virus.

The FDA authorized different trials using emergency procedures. One of the molecular assays adapted to the diagnosis of COVID19 is the transcription-mediated amplification (TMA) assay. We intend to answer the questions that are asked from a laboratory when a new technique is introduced. How does it work? Are your results comparable to gold standard techniques in terms of agreement and limit of detection?

After the systematic review of the literature, an almost perfect concordance between the TMA and the RTPCR was observed, taking into account the values of Cohen's kappa coefficient, positive percentage agreement, negative percentage agreement and global percentage agreement close to $100 \%$.

Regarding the concordance between the results obtained by TMA and the antigenic test, it presents a lower concordance, although it is true that the latter test is only optimal for the detection of SARS-CoV2 in a specific profile of patients. The literature shows that the TMA test has been used to study possible cases of reinfection. After analyzing the results, we concluded in the proven consolidation of TMA for the diagnosis and follow-up of SARS-COV2.

\section{Introduction}

Since the first group of patients suffering from severe pneumonia of unknown origin was identified in December 2019 in Wuhan (China), which was called COVID-19 (Coronavirus Disease-2019) and its causative agent, severe acute respiratory syndrome coronavirus 2 (SARS-CoV-2) to date it has not been more than a year.(Zhu N, et al. 2020). In a matter of a few months, the WHO declared it a pandemic due to its global impact. The large number of infected, many of them serious, collapsed the health systems of most countries (Velavan, T. P et al 2020).

The epidemiological fight, the search for cases and the isolation of the sick were established as one of the main tools to contain the pandemic. To carry out effective isolation, a correct use of laboratory diagnostic tests is necessary. The identification of viral RNA in patients with compatible symptoms has served as the main parameter in the search for positives ( $\mathrm{Li}, \mathrm{Y}$ et al 2020). The most widely used genetic technique has been the real-time polymerase chain reaction (RT-PCR) looking for different genetic targets. Over time, other molecular diagnostic techniques, some more automated, have been adapted to meet the ever increasing demand for COVID diagnostics requests in clinical and microbiological laboratories. One of the techniques adapted to the diagnosis of COVID-19 is the transcription-mediated amplification assay, TMA (Avaniss-Aghajani, E et al. 2020). Laboratories that have diferents techniques (TMA and RT-PCR tests,FIA...) are being forced to use the these tests interchangeably in order to process all the requests 
they receive. Therefore, it is necessary to confirm the concordance in the results between the TMA assay and the RT-PCR assay and antigen test.

Our objective is to carry out a review where the works that present the main characteristics of the TMA technique that show us that it can be or is already a reference test during the pandemic are grouped.

To do this, we are going to define the method of the trial, the agreement in the results between the TMA trial versus the other diagnostic options, as well as its use to study possible reinfections.

With this, we will draw conclusions about it and fill the gap that so far exists in the literature. Laboratory professionals, as well as medical professionals, need the confidence of knowing whether the result matches the patient's health status regardless of whether an RT-PCR assay, TMA assay, or antigen test has been performed.

\section{Materials And Methods}

A search of articles was carried out in order to find data that would allow us to obtain conclusions about our questions: Development of the transcription amplification technique, comparison between TMA versus other diagnostic tests for SARS-CoV-2 detection. Suitability of using TMA as part of a battery of tests to check for possible reinfections, we carry out the following process.

A search was carried out under the terms (Covid - 19) or (SARS-CoV-2) or (2019-nCOV) or (RT-PCR) or (PCR) or (TMA).These search terms were combined together using the Boolean logic "OR", "AND", "NOT" to set logical relationships between terms and finding the right articles. We used the electronic portals PubMed, Google Academic, Web Of Science and SCOPUS. No time filters were added, nor were language or institution or country filters added. An independent review was carried out with the aim of selecting only those who answered our questions. Selecting those who defined the technique, those who compared the TMA test with other diagnostic techniques and the articles that used the TMA test to confirm a reinfection.

Then we carry out a scrining to eliminate duplicate articles in the different search portals.

We performed an independent reading by the authors of the title and abstract to select the articles that fit our criteria. We excluded clinical cases that only spoke of one patient for the comparison section between TMA and other techniques, instead we accepted this criterion for the section on reinfections.

After adding the articles obtained and discarding the repeated jobs in the different search engines, we obtained a total of 505 jobs. Of which only 17 referred to the use of TMA for the diagnosis of SARS-CoV2. Therefore, 488 articles were excluded whose subject matter did not fit our search objectives (mainly they spoke of the application of TMA in the detection of other microorganisms). Of the 17 remaining studies, those that, although they related technique and pathogen, did not provide solid data to extrapolate to our study, were eliminated. 
After the authors' consensus, we introduced a total of 8 articles in the final review, 497 items were excluded.

With the results obtained, contingency tables were made to obtain data such as Cohen's kappa coefficient $(\kappa)$, the positive percent agreement, the negative percent agreement and overall percent agreement.

All the results and the operations carried out to obtain the result of these statistical parameters (when it did not appear explicitly in the original article) were independently verified by the authors.

All discrepancies and doubts about the data obtained, the veracity of the source or the reproducibility of our work were resolved by the agreement of the authors of this paper.

\section{Results}

\section{Transcription mediated amplification assay, fundamentals of the technique.}

Transcription mediated amplification assay (TMA) is a molecular biology methodology based on the detection of nucleic acids. Automated technique that allows solving large workloads in a short time.

In a first reaction (figure 1), the viral RNA comes into contact with a reverse transcriptase and a cDNA chain is formed. This enzyme is followed by a special RNA polymerase that will create multiple RNA amplicons through the DNA strand, which it will use as a template. Each RNA amplicon will perform the process cyclically, exponentially generating a sufficient amount of genetic load to be detected.

\section{Analytical concordance of results of High-Throughput Transcription-mediated amplification (TMA) versus RT-PCR}

Trémeaux et al. make a comparison between three different trials, pitting them one against one. In a first comparison between Aptima ${ }^{\text {TM }}$ (TMA) and Panther Fusion ${ }^{\text {TM }}$ (RT-PCR) assays after performing a contingency table and analyzing the discrepancies (table 1), they obtain a Cohen's kappa coefficient $\mathrm{K}=$ 0.979 , a positive percent agreement $=97.3 \%$, a negative percent agreement $=100 \%$, and an overall percent agreement $=99.0 \%$. When faced with the results obtained by Aptima ${ }^{\mathrm{TM}}$ assays versus MagNA / LC480 (RT-PCR) assays, they obtained the following concordance data, had a Cohen's coefficient $\mathrm{K}=0.945 \mathrm{a}$ positive percent agreement $=98.6 \%$ a negative percent agreement $=96.9 \%$ and an overall percent agreement $=97.5 \%$. Their invalid rated with the Aptima ${ }^{\mathrm{T}}$ assay was $0.5 \%(1 / 200)$.

Gorzalski et al present a comparison between two different trials. In a first comparison between Aptima ${ }^{\text {TM }}$ (TMA) versus Multiplex assay (RT-PCR) ${ }^{\mathrm{T}}$ assays after performing a contingency table and analyzing the discrepancies obtained (table1) a Cohen's kappa coefficient $\mathrm{K}=0.98$, a positive percent agreement $=$ $98.07 \%$, a negative percent agreement $=100 \%$, and an overall percent agreement $=99.1 \%$. Their invalid rated with the Aptima ${ }^{\mathrm{T} M}$ assay was $3.44 \%(4 / 116)$. 
This work also compares the limit of detection (table2), obtaining favorable results to Aptima ${ }^{\mathrm{TM}}$ (TMA). It is capable of detecting small amounts of viral RNA copies in the sample. In front of a concentration of $5.5 \times 10$ e3 genomic RNA copies/m, TMA rectivity has a value of $5 / 5(100 \%)$ versus $0 / 5(0 \%)$ PCR reactivity. The minimum number of copies $/ \mathrm{ml}$ that is capable of detecting the technique Aptima $^{\text {TM }}$ (TMA) in this work is $5.5 \times 10 \mathrm{e} 2$ copies $/ \mathrm{mL}$.(Table $\mathrm{X}$ )

Smith et al. conducted a study comparing three techniques. Two RT-PCR assays: BioFire Defense COVID19 test (BioFire) and the Hologic Panther Fusion SARS-CoV-2 assay (Fusion) and a TMA Aptima ${ }^{\mathrm{TM}}$ assay. In this case, positive consensus results were obtained (when a positive result coincided in at least two of the three techniques) and a negative consensus result (when a negative result coincided in at least two of the three techniques). Then they faced the results obtained by each trial against the consensus results to obtain the concordance data.

In a first comparison (table 1) with Panther Fusion ${ }^{\text {TM }}$ (RT-PCR) and the consensus results obtain a Cohen's kappa coefficient $\mathrm{K}=0.987$, a positive percent agreement $=98.7 \%$, a negative percent agreement $=100 \%$, and an overall percent agreement $=96.64 \%$. When they compared the results obtained by Aptima ${ }^{\mathrm{T}}$ assays versus the consensus results, they obtained the following concordance data, had a Cohen's coefficient $\mathrm{K}=0.947$ a positive percent agreement $=984.7 \%$ a negative percent agreement $=100 \%$ and an overall percent agreement $=97.3 \%$. Finally, the BioFire trial versus the consensus results obtained the following concordance results, Cohen's coefficient $\mathrm{K}=0.987$, a positive percent agreement $=98.7 \%$, a negative percent agreement $=100 \%$ and an overall percent agreement $=99.3 \%$.

The limit of detection of the three techniques reflects limit of detection values of 62.5 copies / $\mathrm{mL}$ (Fusion), 62.5 copies / mL (Aptima) and a lower limit of detection in Biofire with 125 copies / mL.(table 2).

Pham, $\mathrm{J}$ et al. maked a comparison between two different trials, TMA versus Panther Fusion SARS-CoV-2 RT-PCR assay and get a Cohen's kappa coefficient $\mathrm{K}=0.986$, a positive percent agreement $=100 \%$, a negative percent agreement $=98.7 \%$, and an overall percent agreement $=99.3 \%$.

Cordes, A.K et al. comparison three techniques versus consensus results and we obtained Cohen's kappa coefficient $\mathrm{K}=0.986$, a positive percent agreement $=100 \%$, a negative percent agreement $=98.7 \%$, and an overall percent agreement $=99.3 \%$. for Aptima SARS-CoV-2 TMA and LDT E-gene RT-PCR versus the consensus results. In the study Genesig COVID-19 RT-PCR versus the consensus results obtained Cohen's kappa coefficient $\mathrm{K}=0.900$, a positive percent agreement $=87,5 \%$, a negative percent agreement $=100 \%$, and an overall percent agreement $=95.54 \%$.

Analytical concordance of results of High-Throughput Transcription-mediated amplification (TMA) versus Fluorescent immunoassay (FIA) for antigen detection. 
Beck, E. T et al. They compare the results obtained between TMA, versus the results obtained by FIA, a test that will detect the presence of SARS-COV-2 antigen. The overall positive percent agreement (PPA), negative percent agreement (NPA), and total agreement (TA) of the SOFIA test compared to the APTIMA TMA test were $77.0 \%$ (4 7/61) 99.6\% (284/285), and 95.7\% (331/346), respectively (Table 1$)$. In this article. The patients who participate are separated into two groups. PPA of the SOFIA test with the APTIMA TMA test was $82.0 \%(41 / 50)$ for patients evaluated less than 5 days from the onset of symptoms and $54.5 \%(6 / 11)$ for patients that exceeded 5 days from the onset of symptoms. There was also a difference when the percentage of negative agreement (NPA) and the total agreement (TA) between the two groups were compared. The results obtained were $100 \%$ versus $97.3 \%$ (NPA) and $97 \%$ versus $87.5 \%(T A)$ respectively.

\section{Use of High-Throughput Transcription-mediated amplification (TMA) within the battery of SARS-COV-2 detection techniques in the same patient for the study of reinfections.}

Tillett, R. L et al. show the sequence of tests carried out on a patient who presented symptoms compatible with SARS-COV 2 infection for the study of reinfection. The data obtained in the different tests were: positive RT-PCR (April 18), negative TMA (May 9), negative RT-PCR (May 26), positive RT-PCR (June 5) and positive IgG and IgM serology (June 6). Through genomic sequencing of the virus, they conclude that the patient has undergone a reinfection.

Harrington, D et al. present a reinfection by Variant VOC-202012/01 that occurred in a patient from the United Kingdom. In the study they present several positive molecular diagnostic tests (RT-PCR and TMA) in different periods of time, confirming by sequencing that it is a reinfection.

\section{Discussion}

The first observation that catches our attention after conducting the systematic review of the literature on our subject is the small amount of existing studies. We don't have a big amount of works that comparing the results of TMA versus RT-PCR or versus other assays for the diagnosis of SARS-COV-2, which is a handicap at the time of conducting this review, as well as an inherent weakness of our work. We have few studies on which to collect data. This observation also leads us to understand the need to carry out systematic reviews that group the data from the few existing works to facilitate the search for future readers.

After studying the method of the TMA assay, we observed that it is a technique that uses different enzymes and primers to amplify the possible viral genetic material of the sample. With this, enough genetic material is obtained to be detected from a small amount of sample. This fact allows us not to rule out infections in patients with a low viral load. This unique tube and isothermal method, in addition to being an automated method that allows a large amount of work to be handled in a short time, prevents the sample from being contaminated. 
The concordance in the results obtained when facing TMA versus PCR indicates that we are in front of a technique with a good diagnostic performance.

The consensus results obtain a Cohen's kappa coefficient, a positive percent agreement, a negative percent agreement, and an overall percent showed an almost perfect match and correlation. Both the selected articles and other articles in the literature show concordance results similar to those obtained by confronting TMA versus RT-PCR when we confront two different RT-PCR assays.

For example, in the study presented by Craney, A. R (2020) where they compare two high-throughput reverse transcription PCR systems for the detection of severe acute respiratory syndrome coronavirus 2 , they obtain a Cohen's kappa coefficient 0.913 , lower even than those obtained in the works where the RTPCR assay was compared to the TMA assay.

When we compare the TMA test against an antigenic detection test (FIA) we observe a relatively good concordance between both results, but it is true that the TMA has a higher sensitivity than FIA, being more suitable to avoid false negatives.

In this article (Becks et al, 2020), the patients under study are divided into two groups based on the duration of the symptoms. The results are better in agreement in the group of patients with a symptomatic evolution of less than five days. This fact is explained in their comparison since the commercial company of the FIA test recommends its use in patients with a few days of symptomatic evolution.

Reinfections are a controversial circumstance in the evolution of the pandemic. There are a low number of verified reinfections. A reinfection consists of the infection of the same patient after having overcome the first disease. You need different tests that show that the pathogenic agent (in this case the virus) is present in the first disease, then it is not present in the absence of it and is present again in the second disease. The first reported reinfection caused by SARS-COV2 corresponds to a 25-year-old patient from the United States and published by Richard L. Tillett in The Lancet Infectious Diseases. This work, like the other one presented in the review (Harrington, $\mathrm{D}$ et al), demonstrate the need to use different diagnostic tests throughout the clinical course, as well as the suitability of the TMA assay for this function.

The Aptima $^{\text {TM }}$ SARS-CoV-2 TMA assay was given emergency use authorization (Rhoads, $D$ et al 2020) by the United States Food and Drug Administration and European Community due to the pandemic situation.

Based on the data obtained, we can also understand that TMA is an optimal technique to reduce the number of false negatives, since to the aforementioned agreement we must add its high sensitivity, in some cases even greater sensitivity than RT-PCR to the one being compared. It is vitally important to minimize the number of false negatives. An undiagnosed positive person becomes a potential source of contagion. 
In conclusion, if we add that High-Throughput Transcription-mediated amplification presents detection limits similar to RT-PCR or other diagnostic assays, an almost identical clinical performance in addition to being a more automated process becomes a perfect alternative for the molecular diagnosis of SARSCOV-2 when the laboratory sees it necessary or needs it.

\section{Declarations}

\section{Conflict of Interest}

The authors declare that there is no conflict of interest

\section{References}

1. Zhu, N., Zhang, D., Wang, W., Li, X., Yang, B., Song, J., Zhao, X., Huang, B., Shi, W., Lu, R., Niu, P., Zhan, F., Ma, X., Wang, D., Xu, W., Wu, G., Gao, G. and Tan, W., 2020. A Novel Coronavirus from Patients with Pneumonia in China, 2019. New England Journal of Medicine, 382(8), pp.727-733.

2. VVelavan, T. and Meyer, C., 2020. The COVID-19 epidemic. Tropical Medicine \& International Health, 25(3), pp.278-280.

3. Li, Y., Yao, L., Li, J., Chen, L., Song, Y., Cai, Z. and Yang, C., 2020. Stability issues of RT-PCR testing of SARS-CoV-2 for hospitalized patients clinically diagnosed with COVID-19. Journal of Medical Virology, 92(7), pp.903-908.

4. Avaniss-Aghajani, E., Sarkissian, A., Fernando, F. and Avaniss-Aghajani, A., 2020. Validation of the Hologic Aptima Unisex and Multitest Specimen Collection Kits Used for Endocervical and Male Urethral Swab Specimens (Aptima Swabs) for Collection of Samples from SARS-CoV-2-Infected Patients. Journal of Clinical Microbiology, 58(8).

5. Trémeaux, P., Lhomme, S., Abravanel, F., Raymond, S., Mengelle, C., Mansuy, J. and Izopet, J., 2020. Evaluation of the Aptima ${ }^{\mathrm{TM}}$ transcription-mediated amplification assay (Hologic ${ }^{\circledR}$ ) for detecting SARS-CoV-2 in clinical specimens. Journal of Clinical Virology, 129, p.104541.

6. Gorzalski, A., Tian, H., Laverdure, C., Morzunov, S., Verma, S., VanHooser, S. and Pandori, M., 2020. High-Throughput Transcription-mediated amplification on the Hologic Panther is a highly sensitive method of detection for SARS-CoV-2. Journal of Clinical Virology, 129, p.104501.

7. Smith E, Zhen W, Manji R, Schron D, Duong S, Berry GJ. Analytical and Clinical Comparison of Three Nucleic Acid Amplification Tests for SARS-CoV-2 Detection. J Clin Microbiol. 2020 Aug 24;58(9):e01134-20. doi: 10.1128/JCM.01134-20..

8. Rhoads DD, Cherian SS, Roman K, Stempak LM, Schmotzer CL, Sadri N. Comparison of Abbott ID Now, DiaSorin Simplexa, and CDC FDA Emergency Use Authorization Methods for the Detection of SARS-CoV-2 from Nasopharyngeal and Nasal Swabs from Individuals Diagnosed with COVID-19. J Clin Microbiol. 2020 Jul 23;58(8):e00760-20. doi: 10.1128/JCM.00760-20. 
9. Craney AR, Velu PD, Satlin MJ, Fauntleroy KA, Callan K, Robertson A, La Spina M, Lei B, Chen A, Alston T, Rozman A, Loda M, Rennert H, Cushing M, Westblade LF. Comparison of Two HighThroughput Reverse Transcription-PCR Systems for the Detection of Severe Acute Respiratory Syndrome Coronavirus 2. J Clin Microbiol. 2020 Jul 23;58(8):e00890-20. doi: 10.1128/JCM.0089020.

10. Beck ET, Paar W, Fojut L, Serwe J, Jahnke RR. Comparison of the Quidel Sofia SARS FIA Test to the Hologic Aptima SARS-CoV-2 TMA Test for Diagnosis of COVID-19 in Symptomatic Outpatients. J Clin Microbiol. 2021 Jan 21;59(2):e02727-20.

11. Cordes AK, Rehrauer WM, Accola MA, Wölk B, Hilfrich B, Heim A. Fully automated detection and differentiation of pandemic and endemic coronaviruses (NL63, 229E, HKU1, OC43 and SARS-CoV-2) on the Hologic Panther Fusion. J Med Virol. 2020 Dec 22. doi: 10.1002/jmv.26749. Epub ahead of print.

12. Pham J, Meyer S, Nguyen C, Williams A, Hunsicker M, McHardy I, Gendlina I, Goldstein DY, Fox AS, Hudson A, Darby P, Hovey P, Morales J, Mitchell J, Harrington K, Majlessi M, Moberly J, Shah A, Worlock A, Walcher M, Eaton B, Getman D, Clark C. Performance Characteristics of a HighThroughput Automated Transcription-Mediated Amplification Test for SARS-CoV-2 Detection. J Clin Microbiol. 2020 Sep 22;58(10):e01669-20. doi: 10.1128/JCM.01669-20.

13. Tillett RL, Sevinsky JR, Hartley PD, Kerwin H, Crawford N, Gorzalski A, Laverdure C, Verma SC, Rossetto CC, Jackson D, Farrell MJ, Van Hooser S, Pandori M. Genomic evidence for reinfection with SARS-CoV-2: a case study. Lancet Infect Dis. 2021 Jan;21(1):52-58. doi: 10.1016/S14733099(20)30764-7. Epub 2020 Oct 12.

14. Harrington D, Kele B, Pereira S, Couto-Parada X, Riddell A, Forbes S, Dobbie H, Cutino-Moguel T. Confirmed Reinfection with SARS-CoV-2 Variant VOC-202012/01. Clin Infect Dis. 2021 Jan 9:ciab014. doi: 10.1093/cid/ciab014. Epub ahead of print.

\section{Tables}

Table 1. Concordance of qualitative results. 


\begin{tabular}{|c|c|c|c|c|c|c|}
\hline PAPER & Molecular assay & $\mathbb{N}$ & $\mathrm{K}$ & PPA & PNA & $\begin{array}{l}\text { OVER } \\
\text { PNA }\end{array}$ \\
\hline \multirow[t]{2}{*}{$\begin{array}{l}\text { Trémeaux } \\
\text { et al. }\end{array}$} & $\begin{array}{l}\text { Aptima }^{\mathrm{TM}} \text { (TMA) vs Panther Fusion }{ }^{\mathrm{TM}} \\
\text { Aptima }^{\mathrm{TM}} \text { (TMA) vs MagNA / LC480 } \\
\text { MagNA / LC480 vs Panther Fusion }{ }^{\text {тм }}\end{array}$ & 199 & 0.979 & 97.3 & 96.9 & 97.5 \\
\hline & & 200 & 0.956 & 94.5 & 100 & 98.10 \\
\hline $\begin{array}{l}\text { Gorzalski } \\
\text { et al. }\end{array}$ & $\begin{array}{l}\text { Aptima }^{\mathrm{TM}} \text { (TMA) versus Multiplex assay (RT- } \\
\text { PCR) }{ }^{\mathrm{TM}}{ }^{2} \text { (T) }\end{array}$ & 112 & 0.98 & 98.1 & 100 & 99.1 \\
\hline $\begin{array}{l}\text { Smith et } \\
\text { al. }\end{array}$ & $\begin{array}{l}\text { Panther Fusion }{ }^{\mathrm{TM}} \text { (RT-PCR) vs the consensus } \\
\text { results } \\
\text { Aptima }{ }^{\mathrm{TM}} \text { assays versus the consensus } \\
\text { BioFire trial versus the consensus results }\end{array}$ & 149 & 0.987 & 98.7 & 100 & 96.64 \\
\hline & & 150 & 0.987 & 98.7 & 100 & 99.3 \\
\hline $\begin{array}{l}\text { Pham, J } \\
\text { et al. }\end{array}$ & $\begin{array}{l}\text { Aptima SARS-CoV-2 TMA assay versus Panther } \\
\text { Fusion SARS-CoV-2 RT-PCR assay }\end{array}$ & 140 & 0.986 & 100 & 98.7 & 99.3 \\
\hline $\begin{array}{l}\text { Cordes, } \\
\text { A. K }\end{array}$ & $\begin{array}{l}\text { Aptima SARS-CoV-2 TMA to the consensus } \\
\text { results. } \\
\text { LDT E-gene RT-PCR }\end{array}$ & 157 & 0.986 & 100 & 99 & 99.36 \\
\hline & Genesig COVID-19 RT-PCR & 157 & 0.986 & 100 & 99 & 99.36 \\
\hline & & 157 & 0.900 & 87,5 & 100 & 95.54 \\
\hline $\begin{array}{l}\text { Beck, E. } \\
\text { T et al }\end{array}$ & Aptima ${ }^{\mathrm{TM}}$ assays to FIA & 347 & 0.837 & 77.0 & 99.6 & 95.7 \\
\hline
\end{tabular}

K: Cohen's kappa coefficient $\mathrm{K}$, PPA: Positive percent agreement, PNA: Negative percent agreement, OPA: Overall percent agreement.N population participating in the study

Table 2. Analytical Limit of detection comparison. 


\begin{tabular}{|l|l|l|}
\hline PAPER & Molecular assay & Limit Of detection \\
\hline Gorzalski et al. & Aptima ${ }^{\mathrm{TM}}$ (TMA) & $5.5 \times 10 \mathrm{e} 2 \mathrm{copies} / \mathrm{mL}^{*}$ \\
& Taqpath RT-PCR reactivity & $5.5 \times 10 \mathrm{e} 4 \mathrm{copies} / \mathrm{mL}^{*}$ \\
& CDC RT-PCR & $5.5 \times 10 \mathrm{e} 4 \mathrm{copies} / \mathrm{mL}^{*}$ \\
\hline Smith et al. & $\begin{array}{l}\text { Panther Fusion }{ }^{\mathrm{TM}} \text { (RT-PCR) } \\
\text { Aptima }{ }^{\mathrm{TM}}\end{array}$ & $125 \mathrm{copies} \mathrm{/} \mathrm{mL}^{* *}$ \\
& BioFire trial & 62.5 copies $/ \mathrm{mL}^{* *}$ \\
& 62.5 copies $/ \mathrm{mL}^{* *}$ \\
\hline
\end{tabular}

* Concentration of SARS-CoV-2 genomic RNA, BEI Resources, NR-52285.

** The BEI Resources material was provided at a concentration of $4.1 \times 10^{9}$ genome equivalents $(\mathrm{GE}) / \mathrm{ml}$, from which the following serial dilutions were prepared (in $\mathrm{GE} / \mathrm{ml}$ ): $1,000,500,250,125,62.5$, and 31.3.

\section{Abbreviations}

TMA

High-Throughput Transcription-mediated amplification.

RT-PCR

Real-time polymerase chain reaction

SARS-COV-2

severe acute respiratory syndrome coronavirus 2 .

COVID-19

Coronavirus Disease-2019

DNA

Deoxyribonucleic acid.

cDNA

Complementary deoxyribonucleic acid.

RNA

Ribonucleic acid.

FIA

Fluorescent immunoassay

$\mathrm{mL}$

mililiter agreement (PPA), negative percent agreement (NPA), and total agreement (TA)

PPA

Positive percent agreement.

NPA

Negative percent agreement

TA 
Total Agreement

$\mathrm{K}$

Cohen's kappa coefficient

GE

Genome equivalents

Figures

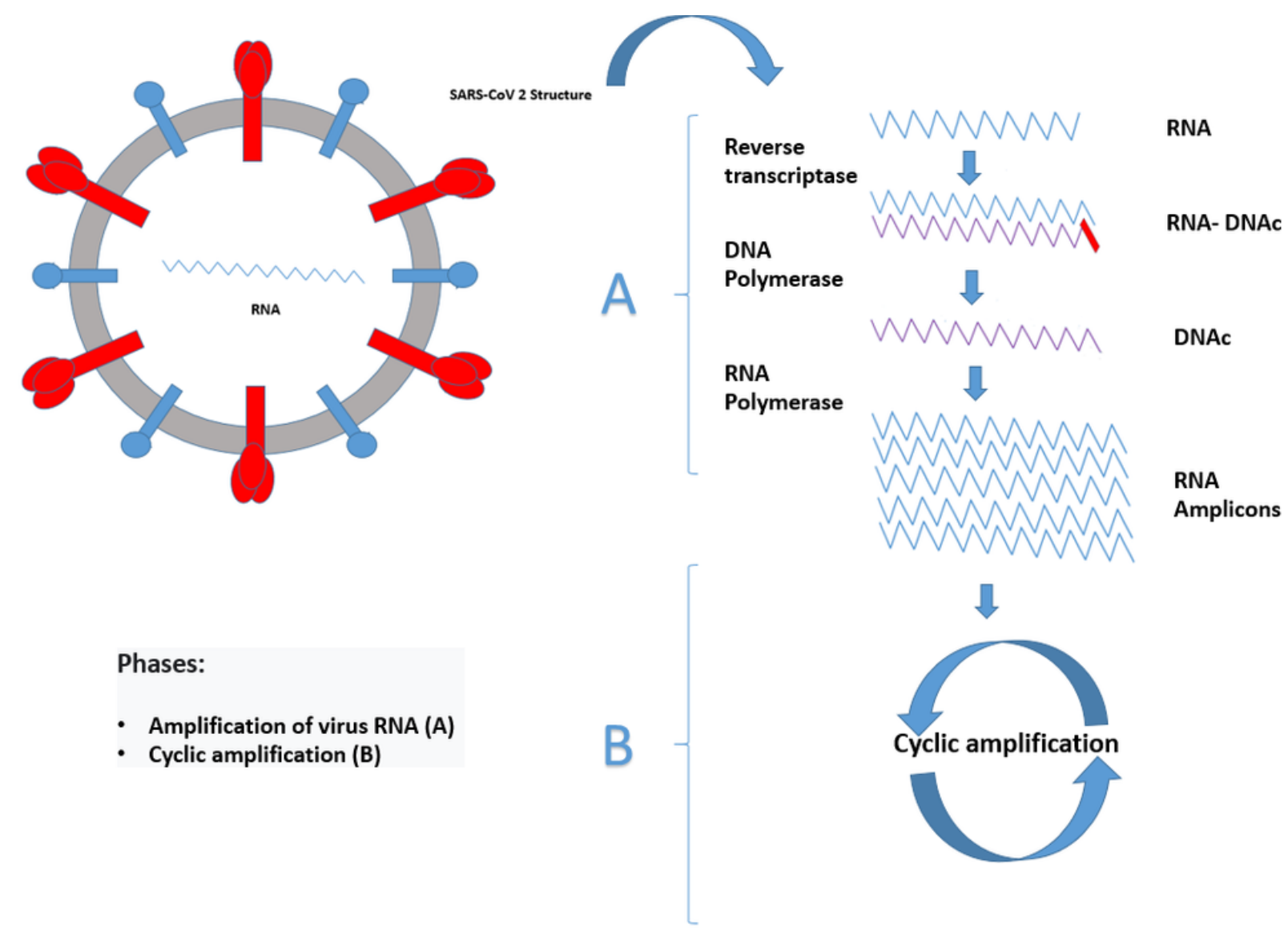

\section{Figure 1}

Fundamentals of the technique. DNA: Deoxyribonucleic acid.RNA Ribonucleic acid. cDNA: Complementary deoxyribonucleic acid. 